\title{
El daño colateral de la atención de la pandemia de COVID-19
} Collateral damage during the attention for COVID-19 pandemic

\author{
Juan M. Abdo-Francis ${ }^{1,2 *}$, Francisco J. Bosques-Padilla ${ }^{3}$, Pedro Gutiérrez-Castrellón ${ }^{4}$ y \\ Sergio R. Sobrino-Cossío $0^{1,5}$ \\ ${ }^{1}$ Centro Avanzado en Endoscopía y Estudios Funcionales, Gástrica, SA de CV, Ciudad de México; ${ }^{2}$ Servicio de Endoscopía, Hospital Ángeles \\ Acoxpa, Ciudad de México; ${ }^{3}$ Facultad de Medicina y Hospital Universitario Dr. José Eleuterio González, Universidad Autónoma de Nuevo León; \\ ${ }^{4}$ Centro de Investigación Traslacional, Hospital General Dr. Manuel Gea González, Ciudad de México; ${ }^{5}$ Servicio de Endoscopia, Hospital Ángeles \\ del Pedregal, Ciudad de México. México
}

\section{Resumen}

A lo largo de la historia, las pandemias han tenido un gran impacto para la humanidad. Las medidas utilizadas para combatirlas causan daño colateral. En la pandemia por COVID-19, las acciones generadas para disminuir la exposición, el número de contagios y la tasa de letalidad conllevan un enfoque en la reducción de la mortalidad, sin embargo el colapso del sistema de salud puede provocar un número aún mayor de muertos. A su vez, tanto el personal médico como los pacientes se ven afectados por la desaceleración económica y el "efecto de la negatividad". En este artículo de revisión se analizaron las diferentes herramientas para el control de la pandemia, su desarrollo en un contexto histórico y como impactan en la estratificación del riesgo para pacientes vulnerables (ancianos, pacientes con enfermedades crónico degenerativas y oncológicos).

Palabras clave: COVID-19. Daño colateral. Impacto en el sistema de salud.

\begin{abstract}
Throughout history, pandemics have had a major impact on humanity. The measures used to combat them cause collateral damage. During the COVID-19 pandemic, the actions taken to reduce the exposure, the number of infections, and the case fatality rate focus on reducing mortality, however, the collapse of the health system can cause an even greater number of deaths. At the same time, both medical personnel and patients are affected by the economic slowdown and the "effect of negativity". In this review article the different tools available for pandemic control, their development in a historical context, and how they may impact risk stratification for vulnerable patients (elderly, patients with chronic degenerative and oncological diseases) were analyzed.
\end{abstract}

Key words: COVID-19. Collateral damage. Health care system impact.

Fecha de recepción: 13-06-2020

Fecha de aceptación: 24-06-2020

E-mail: drjuanmiguelabdo@yahoo.com.mx DOI: $10.24875 / C I R U .20000647$

Cir Cir. 2020;88(6):799-804

Contents available at PubMed www.cirugiaycirujanos.com 0009-7411/@ 2020 Academia Mexicana de Cirugía. Publicado por Permanyer. Este es un artículo open access bajo la licencia CC BY-NC-ND (http://creativecommons.org/licenses/by-nc-nd/4.0/).
} 
«El que lucha contra los monstruos debería tener cuidado de no convertirse en un monstruo. Si miras demasiado al abismo, el abismo te mirará a ti.»

F. Nietzsche (Más allá del bien y del mal)

Las pandemias de gripe de 1918 y de sida fueron devastadoras para la humanidad. En la actualidad, más allá de lo sanitario, vivimos una etapa crítica. Las crisis son cíclicas, raras y pasajeras, y pueden ser explicadas por sus causas. Si se vuelven permanentes, se convertirán en la causa que puede explicar lo demás.

En el año 2020, un nuevo virus quebró bruscamente el orden social, económico, político e ideológico, así como el sentimiento de seguridad dentro de nuestra sociedad. Pareciera que la globalización precipitó que «todo lo sólido se desvanezca en el aire»; esa solidez que nos daba la seguridad ${ }^{1}$.

El término "pandemia» se refiere a que todo el pueblo está incluido. Lo paradójico es que el aislamiento social es parte de la solidaridad.

El ataque a las Torres Gemelas de Nueva York es un clásico ejemplo de "cisne negro". Un evento de esa naturaleza es sorpresivo (para el observador), tiene gran impacto (generalmente negativo) y es impredecible. Aunque los datos estén disponibles, no son contabilizados para mitigar los riesgos. Estos eventos extremos pueden predecirse hasta cierto punto. La pandemia de COVID-19 producida por el SARS-CoV-2 no es un cisne negro, sino más bien un cisne blanco, ya que tuvo un gran impacto y fue predecible y probable? .

Sin embargo, cuando un evento tiene un gran tamaño o magnitud del efecto («un rey») comparte un solo origen («un dragón»), el cual está relacionado con otros dentro del mismo sistema. Esta teoría es conocida como el «rey dragón».

En los sistemas no lineales y complejos, los mecanismos dependen de la retroalimentación positiva, sus puntos de inflexión, bifurcaciones y las transiciones de la fase, que en conjunto tienden a amplificar a niveles extremos la ocurrencia del evento. La comprensión del sistema y el monitoreo de sus dinámicas permitirán predecir su ocurrencia ${ }^{3}$.

Si hacemos afirmaciones sobre lo desconocido con base en las propiedades que asumimos como lo conocido, caeremos en el problema clásico de la inducción: 1) si la probabilidad de ocurrencia de un evento es menor, mayor deberá ser el tamaño de la muestra para hacer inferencias, y 2) cuanto menor es la probabilidad, mayor será el error de la estimación².
Los errores de estimación sobre las probabilidades son importantes cuando se considera un gran impacto. La ciencia trata de poner orden a la confusión mediante la eliminación de la incertidumbre. Se ha considerado que, si tenemos una distribución de probabilidades normal (o gaussiana), entonces contaremos con datos suficientes para hacer inferencias. ¿Pero qué sucede si la distribución no es simétrica? ${ }^{2}$ Aunque nos sorprende la magnitud del efecto, una catástrofe ecológica global o la pandemia por COVID-19 podrían ser consideradas como «rey dragón» (doble bifurcación), en donde los precursores pudieron ser detectados ya que mostraban señales. Otros ejemplos son los accidentes nucleares, la quiebra financiera de los mercados, el desplome de los precios del petróleo, los desastres naturales, etc. ${ }^{2}$.

Una característica de este fenómeno es su retroalimentación positiva. En la dinámica humana, ocurre un comportamiento de rebaño («pastoreo y mafia»). Es decir, el pánico durante una estampida (multitudes, mercados bursátiles, etc.) provocará más pánico, lo que hará que el ganado corra más y más, y así sucesivamente ${ }^{2,4}$.

La toma de decisiones «a toro pasado» es dependiente de los datos con que contamos. En la gestión de los riesgos, el mal uso estadístico y la dependencia de la probabilidad basada en los métodos actuales puede tener graves consecuencias. Cuanto menor es la probabilidad, más consecuente es el impacto del error.

A pesar del gran impacto de los eventos extremos, fallamos al anticiparlos, ya sea por ignorancia o sesgos cognitivos, mala selección del modelo, el mal uso de las distribuciones (gaussiana), lo cual subestimará el efecto. La teoría del «rey dragón» por definición emplea valores atípicos y plantea un reto para predecir el riesgo.

Ante la necesidad de regresar a nuestras actividades diarias y el riesgo de contagio de COVID-19 (no negociable) debemos negociar entre la compensación del riesgo y el rendimiento. Si la predicción «rey dragón» es exitosa para adaptarnos a las nuevas circunstancias, podremos defender el sistema (ganancia), mediante el diseño de resiliencia y el monitoreo de riesgos basado en la frecuencia y la gravedad de la enfermedad para la toma de decisiones.

\section{Las medidas implementadas y sus consecuencias}

Las enfermedades infecciosas, a lo largo de nuestra historia, han sido la mayor causa de mortalidad. El 
objetivo de implementar medidas duras e inmediatas durante una pandemia es evitar un pico tardío de infecciones. El no adoptarlas provocará millones de infectados, muchos de ellos hospitalizados, los cuales requerirán cuidados intensivos o morirán por el colapso del sistema de salud. Las medidas pueden implementarse a un costo razonable para la sociedad ${ }^{5}$.

Los gobiernos eligieron una de las siguientes opciones para enfrentar la pandemia, con base a sus condiciones económicas y políticas, y considerando las repercusiones económicas ${ }^{5,6}$ :

- No hacer nada. El riesgo de esta estrategia es tener un mayor número de infectados, el colapso del sistema de salud, una mayor tasa de letalidad y una mortalidad de unos 10 millones de personas. Una de las presunciones de esta estrategia es que se obtendría una inmunidad de rebaño.

- Estrategia de mitigación. No se trata de contener la pandemia, sino de aplanar levemente la curva. Está basada en el distanciamiento social mientras se descubre una vacuna, y esperando que el virus no mutará demasiado.

- Estrategia de supresión. Es la aplicación de medidas duras para el control rápido de la epidemia. Entre ellas, el distanciamiento social, gradual y controlado, y la reanudación posterior de la vida social y económica. Esta estrategia busca ganar tiempo y reducir el número de casos para que no impacte en el sistema de salud.

La última estrategia es descrita como «el martillo», actuar rápido y de manera agresiva, y «la danza», refiriéndose al período entre «el martillo» y la aparición de la vacuna o del tratamiento, ya que las medidas duras no podrán ser permanentes y deberán ser ajustadas, extremando o no el distanciamiento social. La mayoría hizo lo correcto: "el martillo» fue la decisión correcta, ya que dio tiempo de reducir la expansión de la epidemia y descubrir qué hacer durante "la danza» para evitar un segundo brote ${ }^{6}$.

El modelado matemático sobre las enfermedades infecciosas puede mejorar la predicción: ¿qué condiciones son necesarias para el brote?, ¿qué población se infectará?, ¿es protectora la vacunación? La cladística, bajo la teoría de la evolución, es el mejor abordaje, ya que considera las variantes genéticas y su interacción con el ambiente para entender el comportamiento. Con ello podemos predecir los efectos de una segunda o futuras oleadas, clasificar organismos y predecir lo que aún no se ha observado ${ }^{7}$.

\section{El daño colateral}

Las estrategias contra la epidemia de COVID-19 tienen como objetivos disminuir la exposición, el número de contagios, la tasa de letalidad y el colapso del sistema de salud. En este escenario, las cifras de mortalidad están centradas en muertes directas por coronavirus. Sin embargo, si colapsa el sistema de salud, muchos morirán por otras enfermedades.

Además de la desaceleración de la actividad económica, que puede tener consecuencias graves, se ha observado un incremento de la ansiedad y la depresión como una consecuencia del «efecto de la negatividad»; es decir, nuestro cerebro está bajo un bombardeo mediático continuo y genera una atmósfera de catastrofismo. Esta situación puede detonar problemas psiquiátricos no evidenciados, controlados o no diagnosticados, así como una mayor agresividad, intentos de suicidio, consumo de sustancias o drogas, y aislamiento.

Un editorial alertó sobre un tsunami de daños colaterales por la COVID-198. El artículo enfatizó que es imprescindible minimizar el daño en los pacientes vulnerables y en aquellos con mayor riesgo de muerte asociado a las enfermedades crónicas no transmisibles. ¿Pero qué sucederá si todo el sistema de salud colapsa por los pacientes con coronavirus? Seguramente, otros también morirán por otras enfermedades.

Uno de los mayores problemas mundiales es la prevalencia de la obesidad (índice de masa corporal $>30 \mathrm{~kg} / \mathrm{m}^{2}$ ), que en la población americana es del $33 \%$ y en México del $32 \%$ en mayores de 20 años, y que además ha sido asociada con el síndrome metabólico $0^{9,10}$.

En los países con ingresos bajos y medios, las enfermedades crónicas no transmisibles (cáncer, cardiovasculares, accidentes cerebrovasculares y diabetes) superan a las infecciosas como causa de muerte. Esta transición epidemiológica y demográfica ha sido impulsada por el desarrollo económico (índice de desarrollo humano), atribuible al ambiente $u$ "occidentalización». Esto es una consecuencia de los cambios demográficos (envejecimiento y crecimiento de la población), la exposición a factores de riesgo como el fumar y el consumo de alcohol, y la disminución de las infecciones como causa de muerte infantil ${ }^{11}$.

La decisión de aplazar o reprogramar, por ejemplo, las colonoscopias durante la pandemia de COVID-19 en sujetos con una prueba inmunoquímica de sangre oculta en heces positiva, se basa en: 1) la selección de pacientes (urgente/electiva) basada en la 
morbimortalidad por la enfermedad de base y por la infección por COVID-19, y 2) la disminución del riesgo (pacientes y personal de la salud) mediante el uso del equipo de protección personal. Los programas de tamizaje y las colonoscopias programadas de sujetos con una prueba inmunoquímica de sangre oculta en heces positiva (fuera de los programas nacionales) fueron considerados de baja prioridad (postergables) $^{12}$.

¿Cuál será el tamaño del efecto de aplazar las colonoscopias por la pandemia? ¿Cómo será el impacto en la carga asistencial? ¿Influirá negativamente en los hallazgos de la colonoscopia? La reanudación de las actividades endoscópicas será compleja y representará un verdadero reto, ya que es difícil poner una fecha probable para volver a la normalidad. La reprogramación y el postergamiento de la colonoscopia seguramente impactará, de manera negativa, en los hallazgos y en la carga asistencial si los programas no son reanudados a la brevedad. Sin embargo, la reanudación de las actividades endoscópicas será compleja y es difícil ponerle una fecha. Además, habrá que considerar que esta población (mayores de 50 años), que acude en mayor porcentaje a estos procedimientos, tiene mayor riesgo de comorbilidad.

Por otra parte, existen otros factores que pueden incrementar la mortalidad asociada a neoplasia e infección, como son el incremento de la pirámide poblacional y las enfermedades crónicas no transmisibles. En Uruguay, por ejemplo, donde hay una alta prevalencia de cáncer colorrectal, la prevalencia de sobrepeso $\left(>25 \mathrm{~kg} / \mathrm{m}^{2}\right)$ y de obesidad $\left(30 \mathrm{~kg} / \mathrm{m}^{2}\right)$ reportada es de 64.5 [59.8-69.2] y 28.9 [23.7-34.4], respectivamente, con un incremento del riesgo ${ }^{13}$. Además, el riesgo de mortalidad por enfermedad cardiovascular entre los 50 y los 79 años es del $41 \%{ }^{14}$.

La pandemia plantea dos problemas fundamentales en relación con la seguridad en el paciente oncológico: 1) la visita hospitalaria incrementa la exposición a la infección, y 2) los tratamientos pueden predisponer a efectos más nocivos por COVID-19. ¿Deben arriesgarse a exponerse al SARS-CoV-2 para recibir tratamiento contra el cáncer? La evidencia disponible es limitada, pero sugiere que los síntomas de la COVID-19 son probablemente más graves en los pacientes con cáncer que en aquellos sin cáncer.

Para garantizar la seguridad y disminuir los riesgos de transmisión, los sistemas de salud recomiendan en la mayoría de los casos posponer o aplazar los tratamientos. Sin embargo, con más nuevos casos de COVID-19 es urgente priorizar el manejo. Existe evidencia de que el aplazamiento puede afectar negativamente los resultados. Por ejemplo, existe un aumento del $16 \%$ en el riesgo de muerte por cada mes de retraso de la radioterapia para pacientes con cáncer de cabeza y cuello (relación de riesgo [RR]: 1.16; intervalo de confianza del 95\% [IC 95\%]: 1.02-1.32) ${ }^{15}$. Y postergar la quimioterapia en el cáncer colorrectal (hazard ratio: 1.14; IC 95\%: 1.10-1.17 por 4 semanas) y el cáncer de mama (RR: 1.08; IC 95\%: 1.01-1.15 por 4 semanas) está asociado a menor supervivencia ${ }^{16,17}$.

Posponer los tratamientos contra el cáncer podría conllevar riesgos, aunque estos deberán considerarse según la magnitud de los beneficios potenciales, el impacto de los tiempos de espera en los resultados y las prioridades competitivas del paciente y del sistema. A lo largo de la pandemia, será importante apoyar el bienestar emocional del paciente (apoyo psicosocial). Las decisiones se basarán en el principio de la precaución, prioridad basada en la evidencia del triaje y la fluidez para reconocer cómo cambian los contextos locales ${ }^{15}$.

Para los sistemas de salud que anticipan casos de COVID-19, pero sin casos confirmados, la continuidad en la mayoría de los tratamientos, mientras se considera posponer aquellos sin efectos adversos anticipados sobre la respuesta oncológica (carcinomas de células basales o cánceres de próstata de bajo riesgo), es razonable. Aplazar las citas de seguimiento durante meses parece razonable para la mayoría de los pacientes bajo estas circunstancias. Algunas visitas hospitalarias podrían reemplazarse con evaluaciones «virtuales» (videoconferencia o teléfono). Este enfoque busca equilibrar los resultados (paciente) y la capacidad del sistema cuando las cuarentenas, los aislamientos y las hospitalizaciones anticipadas afecten la capacidad de brindar atención para el cáncer. Estas medidas garantizan la seguridad del paciente al reducir los riesgos de transmisión a una población vulnerable ${ }^{15}$.

La falta de acceso a la atención médica o el cierre de las instalaciones, las dificultades económicas, las consultas, los tratamientos y los procedimientos perdidos o retrasados, la permanencia domiciliaria por órdenes expresas, y el miedo a contagiarse, son causas del daño colateral en la progresión (exacerbación) de las enfermedades crónicas no transmisibles y la mortalidad prevenible. Si no anticipamos las consecuencias de esta nueva curva, fallaremos irremediablemente al desatender, ignorar, omitir o diferir los programas (difusión de la salud, prevención, 
detección oportuna, diagnóstico y tratamiento) que atienden las enfermedades crónicas no transmisibles, lo cual tendrá un impacto negativo en la mortalidad y en la carga asistencial del sistema.

En los Estados Unidos de América, la probabilidad de infección por SARS-CoV-2 es de un $75-80 \%$, con una mortalidad estimada del 5\% (aproximadamente 10 millones de personas) ante la propagación del virus. Cada año hay 4 millones de personas internadas en las unidades de cuidados intensivos y 500,000 (en torno al $13 \%$ ) de ellas mueren. La mortalidad podría ser cercana al $80 \%$ sin el acceso a las unidades de cuidados intensivos como daño colateral. Es imprescindible evitar el colapso del sistema de salud estableciendo la consulta virtual ${ }^{8}$.

\section{Estratificación del riesgo en las enfermedades crónicas}

Los pacientes con enfermedades crónicas no transmisibles representan un grupo muy heterogéneo con diferentes niveles de riesgo. Debemos evaluar de manera especial a aquellos que no han recibido atención (diagnóstico) o que han interrumpido el tratamiento, ya que aumenta considerablemente el riesgo de hospitalización y muerte por COVID-19 o por comorbilidad. Una vez identificado el riesgo hay que considerar los factores socioeconómicos (ingreso, ahorro, empleo/desempleo, dificultades económicas) y clínicos (perfil o gravedad, control de la enfermedad, apego al tratamiento, requerimiento de laboratorio o de gabinete).

La analítica avanzada y la inteligencia artificial permiten la estratificación del riesgo teniendo en cuenta factores socioeconómicos y clínicos. Estas medidas pueden ser útiles para mantener sanos, seguros y fuera del hospital a los pacientes vulnerables ${ }^{8}$.

Debemos asegurar la consulta programada a distancia (electrónicas especializadas) y las visitas domiciliarias para asegurar el apego y los regímenes de tratamiento, además de implementar el suministro de los medicamentos con un monitoreo remoto.

El acceso a los cuidados paliativos esenciales al final de la vida se limitará durante la pandemia. El aislamiento y el sufrimiento aumentarán en los pacientes de cuidados paliativos y en aquellos que se encuentran en duelo. Los proveedores de cuidados, incluidos los centros privados, requerirán recursos humanos y financieros adicionales. La capacitación en línea está disponible para proporcionar algunos tipos de cuidados. Los teléfonos inteligentes y la telemedicina pueden facilitar las actividades en el hogar respaldadas por profesionales de la salud y los voluntarios sin el contacto físico ${ }^{18}$.

Un estudio realizado en California reportó una menor incidencia (eventos por 100,000 persona-semanas) de hospitalización aguda por infarto al miocardio después de los primeros casos de COVID-19 al comparar dos periodos (1 enero a 3 marzo vs. 4 marzo a 14 abril); estos hallazgos fueron similares a los reportados en Italia ${ }^{19}$.

Existen procedimientos que no pueden diferirse porque se incrementa el riesgo de mortalidad, como el reemplazo de válvula aórtica en pacientes con insuficiencia cardiaca, en quienes la tasa de mortalidad es del 23\% (a 6 meses) si se retrasa la intervención.

Aunque el tratamiento quirúrgico de la enfermedad no relacionada con la COVID-19 es necesario, los procedimientos no urgentes han sido reducidos debido a la necesidad de redistribuir los recursos de atención médica para satisfacer las necesidades médicas masivas proyectadas por la COVID-19. La decisión de realizar procedimientos necesarios y urgentes requiere la incorporación de factores que deberán ser evaluados (limitaciones de recursos, riesgo de transmisión del SARS-CoV-2 para proveedores y pacientes, etc.).

Los autores describieron un sistema de puntuación que integra sistemáticamente los factores para facilitar la toma de decisiones y la clasificación de los procedimientos, y sopesa el riesgo individual contra la necesidad ética de optimizar las preocupaciones de la salud pública. La utilidad práctica es que la estrategia permitió identificar el riesgo, detectar el impacto del retraso del procedimiento sobre el tratamiento exitoso de la enfermedad o de los riesgos técnicos y la seguridad al diferirlo ${ }^{20}$.

Estas necesidades insatisfechas representan un grave riesgo para quienes son los más vulnerables desde el punto vista clínico en nuestra sociedad. La atención de la pandemia de COVID-19 debe buscar el menor daño colateral posible. Concluimos con una frase sobre la experiencia de la modernidad: «Todo lo sólido se desvanece en el aire; todo lo sagrado es profanado, y los hombres, al fin, se ven forzados a considerar serenamente sus condiciones de existencia y sus relaciones recíprocas».

\section{Conflicto de intereses}

Los autores declaran que no existe conflicto de intereses. 


\section{Bibliografía}

1. Berman M. Todo los sólido se desvanece en el aire. La experiencia de la modernidad. 2. ${ }^{a}$ ed. Ciudad de México: Editorial Siglo XXI; 2011.

2. Taleb NN. The Black Swan: the impact of the highly improbable.2nd ed. London: Penguin; 2010.

3. Sornette D, Ouillon G. Dragon-kings: mechanisms, statistical methods and empirical evidence. The European Physical Journal Special Topics. 2012;205.1:1-26.

4. Sornette D. Dragon-Kings, Black Swans and the prediction of crises. International Journal of Terraspace Science and Engineering. 2009;1:1-17.

5. Pueyo T. Coronavirus: The Hammer and the Dance. Consultado: 19 marzo 2020. Disponible en: https://medium.com/@tomaspueyo/coronavirus-the-hammer-and-the-dance-be9337092b56.

6. Pueyo T. Coronavirus: Why you must act now. Politicians, community leaders and business leaders: What should you do and when. Medium. Consultado: 29 abril 2020. Disponible en: https://medium.com/@tomaspueyo/coronavirus-act-today-or-people-will-die-f4d3d9cd99ca.

7. Diaz-Badillo A, Ramirez-pfeiffer C, Lopez-Alvarenga JC. Redes sociales, aprendizaje automatizado y cladística en tiempos del COVID-19. CIRCIR. 2020;88(4):395-8.

8. Reisman L. A tsunami of Covid-19 collateral damage looms. Here's how we save the most lives. Consultado: 19 marzo 2020. Disponible en: https://medcitynews. com/2020/05/a-tsunami-of-covid-19-collateral-damage-looms-heres-how-wesave-the-most-lives/.

9. Reding-Bernal A, Sánchez-Pedraza V, Moreno-Macías H, Sobrino-Cossío S, Tejero- Barrera ME, Burguete-García Al, et al. Heritability and genetic correlation between GERD symptoms severity, metabolic syndrome, and inflammation markers in families living in Mexico City. PLoS One. 2017;12:e0178815.

10. Gutiérrez JP, Rivera-Dommarco J, Shamah-Levy T, Villalpando-Hernández S, Franco A, Cuevas-Nasu L, et al. Encuesta Nacional de Salud y Nutrición 2012. Resultados Nacionales. Cuernavaca, México: Instituto Nacional de Salud Pública; 2012. Disponible en: http://ensanut.insp.mx/ informes/ ENSANUT2012ResultadosNacionales.pdf
11. Forman D, Sierra MS. Cancer in Central and South America: introduction. Cancer Epidemiol. 2016;44(Suppl 1):S3-S10.

12. Hernandez V, Cubiella J, Gonzalez-Mao MC, Iglesias F, Rivera C, Iglesias $\mathrm{MB}$, et al. Fecal immunochemical test accuracy in average-risk colorectal cancer screening. World J Gastroenterol. 2014;20:1038-47.

13. Kaufmann R, Pontet-Ubal N. The challenge of tackling the obesity economic burden: the case of Uruguay. Emerald Open Res. 2019;1:11.

14. Lloyd-Sherlock P, Ebrahim S, Martinez R, McKee M, Ordunez P. Reducing the cardiovascular disease burden for people of all ages in the Americas region: analysis of mortality data, 2000-15. Lancet Glob Health. 2019;7:e604-12.

15. Chen Z, King W, Pearcey R, Kerba M, Mackillop WJ. The relationship between waiting time for radiotherapy and clinical outcomes: a systematic review of the literature. Radiother Oncol. 2008;87:3-16.

16. Biagi JJ, Raphael MJ, Mackillop WJ, Kong W, KIng WD, Booth CM. Association between time to initiation of adjuvant chemotherapy and survival in colorectal cancer: a systematic review and meta-analysis. JAMA. 2011;305:2335-42.

17. Raphael MJ, Biagi JJ, KOng W, Mates M, Booth CM, Mackillop WJ. The relationship between time to initiation of adjuvant chemotherapy and survival in breast cancer: a systematic review and meta-analysis. Breast Cancer Res. Treat. 2016:160:17-28.

18. Radbruch L, Knaul FM, de Lima L, de Joncheere C, Bhadelia A. The key role of palliative care in response to the COVID-19 tsunami of suffering. Lancet. 2020;395:1467-9.

19. Graff A. California reports its first coronavirus death. San Francisco Chronicle. March 4, 2020. De Filippo O, D'Ascenzo F, Angelini F, Bocchino PP, Conrotto F, Saglietto A, et al. Reduced rate of hospital admissions for ACS during Covid-19 outbreak in northern Italy. N Engl J Med. 2020;383:88-9.

20. Prachand VN, Milner R, Angelos P, Posner MC, Fung JJ, Agrawal N, et al. Medically necessary, time-sensitive procedures: scoring system to ethically and efficiently manage resource scarcity and provider risk during the COVID-19 pandemic. J Am Coll Surg 2020 Apr 9;S10727515(20)30317-3. doi: 10.1016/j.jamcollsurg.2020.04.011. Online ahead of print. 